Pacific

Journal of

Mathematics

A NOTE ON THE GAUSS MAP OF COMPLETE NONORIENTABLE MINIMAL SURFACES

Francisco J. LóPEZ and Francisco Martín 


\title{
A NOTE ON THE GAUSS MAP OF COMPLETE NONORIENTABLE MINIMAL SURFACES
}

\author{
Francisco J. López AND Francisco Martín
}

\begin{abstract}
We construct complete nonorientable minimal surfaces whose Gauss map omits two points of $\mathbb{R P}^{2}$. This result proves that Fujimoto's theorem is sharp in nonorientable case.
\end{abstract}

\section{Introduction and Preliminaries.}

The study of the Gauss map of complete orientable minimal surfaces in $\mathbb{R}^{3}$ has achieved many important advances and also has given rise to many problems in recent decades. The most interesting question is to determine the size of the spherical image of such a surface under its Gauss map.

R. Osserman was the person who started the systematic development of this theory, and so, in 1961 he proved that the set omitted by the image of a complete non flat orientable minimal surface by the Gauss map has logarithmic capacity zero. In $1981 \mathrm{~F}$. Xavier [12] proved that this set covers the sphere except six values at the most, and finally in $1988 \mathrm{H}$. Fujimoto $[3,4]$ obtained the best possible theorem, and proved that the number of exceptional values of the Gauss map is four at the most. An interesting extension of Fujimoto's theorem was proved in 1990 by X. Mo and R. Osserman [7]. They showed that if the Gauss map of a complete orientable minimal surface takes on five distinct values only a finite number of times, then the surface has finite total curvature.

There are many kinds of complete orientable minimal surfaces whose Gauss map omits four points of the sphere. Among these examples we emphasize the classical Scherk's doubly periodic surface and those described by K. Voss in [10] (see also [8]). The first author of this paper in [5] constructs orientable examples with non trivial topology.

Under the additional hypothesis of finite total curvature, R. Osserman [9] proved that the number of exceptional values is three at the most.

In the nonorientable case, the Gauss map of the two sheeted orientable covering surface induces, in a natural way, a generalized Gauss map from the nonorientable surface on the projective plane. From Fujimoto's theorem applied to the two sheeted orientable covering, this generalized Gauss map omits two points of $\mathbb{R P}^{2}$ at the most.

It left open the following questions: 
1) Are there complete nonorientable minimal surfaces in $\mathbb{R}^{3}$ whose generalized Gauss map omits two points of $\mathbb{R P}^{2}$ ?

2) Are there complete non flat orientable minimal surfaces in $\mathbb{R}^{3}$ with finite total curvature whose Gauss map omits three points of $\mathbb{S}^{2}$ ?

3) Are there complete nonorientable minimal surfaces in $\mathbb{R}^{3}$ with finite total curvature whose generalized Gauss map omits one point of $\mathbb{R P}^{2}$ ?

Concerning the second problem, A. Weitsman and F. Xavier in [11] and Y. Fang in [1] obtained nonexistence results, provided that the absolute value of the total curvature is less than or equal to $16 \pi$ and $20 \pi$, respectively.

In this paper we give an affirmative answer to the first question, and prove:

Theorem. There are complete nonorientable minimal surfaces in $\mathbb{R}^{3}$ whose generalized Gauss map omits two points of the projective plane.

Our method of construction is somewhat explicit and very simple, and it is based on a more elaborate use of the Voss technique.

Finally, we briefly summarize some of the basic facts we will need in this paper.

Let $X: M \longrightarrow \mathbb{R}^{3}$ be a minimal immersion of a surface $M$ in three dimensional Euclidean space. Using isothermal parameters, $M$ has in a natural way a conformal structure. When $M$ is orientable, we label $(g, \eta)$ as the Weierstrass data of $X$. Remember that the stereographic projection $g$ of the Gauss map of $X$ is a meromorphic function on $M$, and $\eta$ is a holomorphic 1-form on $M$.

Moreover,

$$
X=\text { Real } \int\left(\Phi_{1}, \Phi_{2}, \Phi_{3}\right),
$$

where $\Phi_{1}=\frac{1}{2} \eta\left(1-g^{2}\right), \Phi_{2}=\frac{i}{2} \eta\left(1+g^{2}\right), \Phi_{3}=\eta g$ are holomorphic 1-forms on $M$ satisfying:

$$
\left(\sum_{j=1}^{3}\left|\Phi_{j}\right|^{2}\right)(P) \neq 0, \quad \forall P \in M .
$$

In particular, $\Phi_{j}, j=1,2,3$, have no real periods on $M$. Furthermore, the Riemannian metric $d s^{2}$ induced by $X$ on $M$ is given by:

$$
d s^{2}=\sum_{j=1}^{3}\left|\Phi_{j}\right|^{2} .
$$

For more details see [8].

Consider now $X^{\prime}: M^{\prime} \longrightarrow \mathbb{R}^{3}$ a conformal minimal immersion of a nonorientable surface $M^{\prime}$ in $\mathbb{R}^{3}$. Let $\pi_{0}: M \rightarrow M^{\prime}, I: M \rightarrow M$ denote the conformal oriented two sheeted covering of $M^{\prime}$ and the antiholomorphic order two deck transformation for this covering, respectively. 
If $(g, \eta)$ represents the Weierstrass data of $X=X^{\prime} \circ \pi_{0}$, then it is not hard to deduce that:

$$
I^{*}\left(\Phi_{j}\right)=\overline{\Phi_{j}}, \quad j=1,2,3 .
$$

In particular, $g \circ I=I_{0} \circ g$, where $I_{0}(z)=-1 / \bar{z}$, and so there is a unique map

$$
G: M^{\prime} \longrightarrow \mathbb{R} \mathbb{P}^{2} \equiv \overline{\mathbb{C}} /\left\langle I_{0}\right\rangle
$$

satisfying

$$
G \circ \pi_{0}=g \circ p_{0},
$$

where $p_{0}: \overline{\mathbb{C}} \rightarrow \overline{\mathbb{C}} /\left\langle I_{0}\right\rangle$ is the natural projection. We call $G$ the generalized Gauss map of $X^{\prime}$.

Conversely, given $(M, g, \eta)$ the Weierstrass representation of a minimal immersion $X$ of an orientable surface $M$ in $\mathbb{R}^{3}$, and given $I: M \rightarrow M$ an antiholomorphic involution without fixed points on $M$ satisfying (1), then $X$ induces a minimal immersion $X^{\prime}$ of $M^{\prime}=M /\langle I\rangle$ in $\mathbb{R}^{3}$ such that $X=X^{\prime} \circ \pi_{0}$. For more details see [6].

Finally, denote:

- $\mathbb{D}=\{z \in \mathbb{C}:|z|<1\}$,

- $\mathbb{D}^{*}=\mathbb{D}-\{0\}$,

- for each $R>1, A(R)=\{z \in \mathbb{C}: 1 / R<|z|<R\}$.

Throughout the proof of Theorem 2, we will use the following result:

Theorem 1. Let $M$ be a Riemann surface with holomorphic universal covering space $\mathbb{D}$. Then $M \cong \mathbb{D}, \mathbb{D}^{*}$, or $A(R)$, provided $\Pi_{1}(M)$ is commutative.

The proof of this theorem can be found in [2, Chapter IV].

\section{Main Theorem.}

To obtain the result we have stated in the introduction, we need the following two Lemmas.

Lemma 1. There exist $R>1$ and holomorphic 1 -forms $\Phi_{j}, j=1,2,3$, on $A(R)$ such that:

1) $\Phi_{1}^{2}+\Phi_{2}^{2}+\Phi_{3}^{2} \equiv 0$.

2) $\left|\Phi_{1}\right|^{2}+\left|\Phi_{2}\right|^{2}+\left|\Phi_{3}\right|^{2} \neq 0$.

3) The metric $d s^{2} \stackrel{\text { def }}{=}\left|\Phi_{1}\right|^{2}+\left|\Phi_{2}\right|^{2}+\left|\Phi_{3}\right|^{2}$ is complete.

4) The Gauss map

$$
g=-\frac{\Phi_{1}+i \Phi_{2}}{\Phi_{3}}
$$

omits four points of the Riemann sphere $\overline{\mathbb{C}}$.

5) $I^{*}\left(\Phi_{j}\right)=\overline{\Phi_{j}}, j=1,2,3$, where $I: A(R) \rightarrow A(R)$ is given by $I(z)=$ $-1 / \bar{z}$. 
Proof. Let $\alpha, \beta \in \mathbb{C}^{*}, \alpha \notin\{\beta,-1 / \bar{\beta}\}$, label

$$
M=\overline{\mathbb{C}}-\left\{\alpha, \beta,-\frac{1}{\bar{\alpha}},-\frac{1}{\bar{\beta}}\right\}
$$

and consider the following Weierstrass representation on $M$ :

$$
\widehat{g}=z, \quad \widehat{\eta}=\frac{i d z}{(z-\alpha)(z-\beta)(\bar{\alpha} z+1)(\bar{\beta} z+1)} .
$$

If we define $\widehat{I}: M \rightarrow M, \widehat{I}(z)=-1 / \bar{z}$, then $\widehat{I}$ is an antiholomorphic involution without fixed points, verifying:

$$
\widehat{g} \circ \widehat{I}=-\frac{1}{\widehat{\widehat{g}}}, \quad \widehat{I}^{*}(\widehat{\eta})=-\overline{\widehat{\eta} \widehat{g}^{2}} .
$$

Thus, if we define:

$$
\begin{aligned}
\widehat{\Phi}_{1} & =\frac{1}{2}\left(1-\widehat{g}^{2}\right) \widehat{\eta}, \\
\widehat{\Phi}_{2} & =\frac{i}{2}\left(1+\widehat{g}^{2}\right) \widehat{\eta}, \\
\widehat{\Phi}_{3} & =\widehat{g} \widehat{\eta}
\end{aligned}
$$

then it is obvious, from $(3)$, that $\widehat{I}^{*}\left(\widehat{\Phi}_{j}\right)=\bar{\Phi}_{j}$. Furthermore, these holomorphic 1-forms satisfy:

- $\widehat{\Phi}_{1}^{2}+\widehat{\Phi}_{2}^{2}+\widehat{\Phi}_{3}^{2} \equiv 0$,

- $\left|\widehat{\Phi}_{1}\right|^{2}+\left|\widehat{\Phi}_{2}\right|^{2}+\left|\widehat{\Phi}_{3}\right|^{2} \neq 0$,

- the Riemannian metric $d \widehat{s}^{2}=\left|\widehat{\Phi}_{1}\right|^{2}+\left|\widehat{\Phi}_{2}\right|^{2}+\left|\widehat{\Phi}_{3}\right|^{2}$ is complete in $M$.

On the other hand, the Uniformization Theorem says us that the holomorphic universal covering of $M$ is either $\mathbb{C}$ or the unit disc, $\mathbb{D}$ (see $[\mathbf{2}, \S I V .4]$ ). However, $\mathbb{C}$ is the conformal covering of only two noncompact Riemann surfaces: $\mathbb{C}$ and $\mathbb{C}^{*}$ (see $[\mathbf{2}, \S I V .6]$ ). Thus, the holomorphic universal covering of $M$ is $\mathbb{D}$. We label $\pi: \mathbb{D} \rightarrow M$ as the conformal covering map.

Let $\widetilde{I}$ be a lift of $\widehat{I}$ to $\mathbb{D}$, and denote $\widetilde{\Phi}_{j}=\pi^{*}\left(\widehat{\Phi}_{j}\right), j=1,2,3$. It is clear that $\widetilde{I}^{*}\left(\widetilde{\Phi}_{j}\right)=\overline{\widetilde{\Phi}_{j}}, j=1,2,3$.

Since $\widehat{I}$ is an antiholomorphic involution in $M$ without fixed points, then $\widetilde{I}^{2 k+1}, k \in \mathbb{Z}$, is an antiholomorphic transformation in $\mathbb{D}$ without fixed points too.

Let us see that $\widetilde{I}^{2 k}, k \in \mathbb{Z}^{*}$, has no fixed points in $\mathbb{D}$. Indeed, note that $\widetilde{I}^{2 k}, k \in \mathbb{Z}^{*}$, is a lift of the identity mapping in $M$. Thus, if $\widetilde{I}^{2 k}$ fixes a point of $\mathbb{D}$, we infer that $\widetilde{I}^{2 k}$ is the identity mapping $\mathbf{1}_{\mathbb{D}}$ in $\mathbb{D}$.

Assume that there is $k>0$ such that $\widetilde{I}^{2 k}=\mathbf{1}_{\mathbb{D}}$. Let

$$
k_{0}=\operatorname{Minimum}\left\{k \in \mathbb{N}^{*}: \widetilde{I}^{2 k}=\mathbf{1}_{\mathbb{D}}\right\} \text {, }
$$


and observe that $k_{0}$ is the finite order of $\widetilde{I}^{2}$. It is clear that $k_{0}>1$. Otherwise, $k_{0}=1$ and so there would be antiholomorphic involutions without fixed points in $\mathbb{D}$, which is absurd. Furthermore, from the definition of $k_{0}$, it is obvious that $\widetilde{I}^{2 k}$ has no fixed points, $0<k<k_{0}$.

Therefore, the quotient $\mathbb{D} /\left\langle\widetilde{I}^{2}\right\rangle$ is a Riemann surface with fundamental group isomorphic to $\mathbb{Z}_{k_{0}}$. No such surface exists (see for instance Theorem 1).

This contradiction implies that $\widetilde{I}^{2 k}, k \in \mathbb{Z}^{*}$, has no fixed points and $\left\langle\widetilde{I}^{2}\right\rangle \cong \mathbb{Z}$. In other words, the map

$$
\zeta: \mathbb{D} \longrightarrow \mathbb{D} /\left\langle\widetilde{I}^{2}\right\rangle
$$

is a cyclic conformal covering and the fundamental group of $\mathbb{D} /\left\langle\widetilde{I}^{2}\right\rangle$ is isomorphic to $\mathbb{Z}$.

Using Theorem 1 we deduce that $\mathbb{D} /\left\langle\widetilde{I}^{2}\right\rangle$ is conformally equivalent to either $\mathbb{D}^{*}$ or $A(R)$, for a suitable $R>1$.

The map $\widetilde{I}$ induces on $\mathbb{D} /\left\langle\widetilde{I}^{2}\right\rangle$ an antiholomorphic involution, $I$. Moreover, $\mathbb{D} /\left\langle\widetilde{I}^{2}\right\rangle$ is in a natural way a covering of $M$, and $I$ is projected under this covering map on the original involution $\widehat{I}$ on $M$. Since $\widehat{I}$ has no fixed points in $M$, the same occurs for $I$ in $\mathbb{D} /\left\langle\widetilde{I}^{2}\right\rangle$.

However, any antiholomorphic involution in $\mathbb{D}^{*}$ extends to $\mathbb{D}$, and is the conjugate of a Möbius transformation leaving $\mathbb{D}$ invariant and fixing 0 . In particular, any such map has infinitely many fixed points in $\mathbb{D}$. Hence, we conclude that $\mathbb{D} /\left\langle\widetilde{I}^{2}\right\rangle$ can not be conformally equivalent to $\mathbb{D}^{*}$, i.e., $\mathbb{D} /\left\langle\widetilde{I}^{2}\right\rangle$ is conformally diffeomorphic to $A(R)$, for a suitable $R>1$.

If we look at $I$ as an antiholomorphic involution in $A(R)$, then elementary arguments of complex analysis give that $I(z)=-1 / \bar{z}, \forall z \in A(R)$.

On the other hand, as $\left(\widetilde{I}^{2}\right)^{*}\left(\widetilde{\Phi}_{j}\right)=\widetilde{\Phi}_{j}$, then $\widetilde{\Phi}_{j}$ can be induced in the quotient $\mathbb{D} /\left\langle\widetilde{I}^{2}\right\rangle, j=1,2,3$. The corresponding holomorphic 1-forms on $\mathbb{D} /\left\langle\widetilde{I}^{2}\right\rangle$ are denoted as $\Phi_{1}, \Phi_{2}$, and $\Phi_{3}$, and they obviously satisfy $1,2,3$ and 5 in the lemma statement.

Finally, the meromorphic function

$$
g=-\frac{\Phi_{1}+i \Phi_{2}}{\Phi_{3}}
$$

clearly omits the points $\alpha, \beta,-1 / \bar{\alpha}$, and $-1 / \bar{\beta}$, and 4 holds. This concludes the proof.

Lemma 2. There exists a rational function $f: \overline{\mathbb{C}} \rightarrow \overline{\mathbb{C}}$ satisfying:

1) The only poles of $f$ are 0 and $\infty$.

2) $f \circ I=\bar{f}$.

3) $f(z) \neq 0$, provided that $|z|=1$. 
4) Residue $\left(\frac{f(z)}{z} d z, 0\right)=0$.

Proof. Define

$$
f(z)=\frac{\left(z-m_{1}\right)\left(z-m_{2}\right)\left(m_{1} z+1\right)\left(m_{2} z+1\right)}{z^{2}},
$$

where $m_{1}, m_{2} \in \mathbb{R}$.

We have

$$
\text { Residue }\left(\frac{f(z)}{z} d z, 0\right)=\left(1-m_{1}^{2}\right)\left(1-m_{2}^{2}\right)-2 m_{1} m_{2} .
$$

The choice $m_{1}=2$ and $m_{2}=\frac{2+\sqrt{13}}{3}$ completes the proof.

Now we are able to prove the main result of this paper.

Theorem 2. There exist complete nonorientable minimal surfaces in $\mathbb{R}^{3}$ whose generalized Gauss map omits two points of $\mathbb{R P}^{2}$.

Proof. Take $A(R), \Phi_{1}, \Phi_{2}$, and $\Phi_{3}$ as in Lemma 1 , and $f$ as in Lemma 2 . Put

and write

$$
\Phi_{j}=\varphi_{j}(z) \frac{d z}{z}
$$

$$
\varphi_{j}(z)=a_{j 0}+\sum_{n>0}\left(a_{j n} z^{n}+(-1)^{n+1} \overline{a_{j n}} z^{-n}\right), \quad a_{j 0} \in i \mathbb{R},
$$

the Laurent series expansion of $\varphi_{j}, j=1,2,3$.

Observe that

$$
f(z)=\sum_{n=1}^{m}\left(b_{n} z^{n}+(-1)^{n} \overline{b_{n}} z^{-n}\right),
$$

where $m \in \mathbb{N}^{*}$. Let $k \in \mathbb{N}, k$ odd, $k>m$, and notice that:

Residue $\left(\left[\sum_{n>0}\left(a_{j n} z^{k n}+(-1)^{n+1} \overline{a_{j n}} z^{-k n}\right)\right] f(z) \frac{d z}{z}, 0\right)=0, \quad j=1,2,3$.

Furthermore, it is obvious from Lemma 2

$$
\text { Residue }\left(a_{j 0} f(z) \frac{d z}{z}, 0\right)=0, \quad j=1,2,3 .
$$

Consider the covering $T_{k}: A(\sqrt[k]{R}) \rightarrow A(R), T_{k}(z)=z^{k}$, and define the holomorphic 1-forms on $A(\sqrt[k]{R})$ :

$$
\Psi_{j}=f(z) T_{k}^{*}\left(\Phi_{j}\right)=k f(z) \varphi_{j}\left(z^{k}\right) \frac{d z}{z}, \quad j=1,2,3 .
$$

Taking into account (4) and (5), we deduce that $\Psi_{j}$ is exact, $j=1,2,3$. 
Moreover, it is clear that:

$$
\sum_{j=1}^{3} \Psi_{j}^{2} \equiv 0
$$

and since $k$ is odd,

$$
I^{*}\left(\Psi_{1}, \Psi_{2}, \Psi_{3}\right)=\left(\overline{\Psi_{1}}, \overline{\Psi_{2}}, \overline{\Psi_{3}}\right),
$$

where $I: A(\sqrt[k]{R}) \rightarrow A(\sqrt[k]{R})$ is the lift of the former involution in $A(R)$, that keeps being the map $I(z)=-1 / \bar{z}$.

Note that $\lim _{k \rightarrow \infty} \sqrt[k]{R}=1$, and remember that the zeroes of $f$ are not in $\mathbb{S}^{1}$. Then, taking $k$ large enough, we can guarantee that $f$ never vanishes in the closure of $A(\sqrt[k]{R})$. So, as the only poles of $f$ are 0 and $\infty$, there exist $c>1$ such that

$$
\frac{1}{c}<|f(z)|<c, \quad \forall z \in A(\sqrt[k]{R}) .
$$

Therefore, $\sum_{j=1}^{3}\left|\Psi_{j}\right|^{2} \neq 0$, and if we define $d s_{0}^{2}=\left|\Psi_{1}\right|^{2}+\left|\Psi_{2}\right|^{2}+\left|\Psi_{3}\right|^{2}$, one has:

$$
\frac{1}{c^{2}} T_{k}^{*}\left(d s^{2}\right) \leq d s_{0}^{2} \leq c^{2} T_{k}^{*}\left(d s^{2}\right) .
$$

Since $d s^{2}$ is complete, the same occurs for the metrics $T_{k}^{*}\left(d s^{2}\right)$ and $d s_{0}^{2}$.

Summarizing, the minimal immersion

$$
\begin{gathered}
X: A(\sqrt[k]{R}) \longrightarrow \mathbb{R}^{3}, \\
X(z)=\operatorname{Real}\left(\int_{1}^{z}\left(\Psi_{1}, \Psi_{2}, \Psi_{3}\right)\right),
\end{gathered}
$$

is well defined, complete, and its Gauss map $g \circ T_{k}$ omits four points of $\overline{\mathbb{C}}$.

From (6), $X$ induces a minimal immersion of the Möbius strip $A(\sqrt[k]{R}) /\langle I\rangle$ in $\mathbb{R}^{3}$, and so the Theorem is proved.

\section{References}

[1] Yi Fang, On the Gauss map of complete minimal surfaces with finite total curvature, Indiana Math. J., 42(4) (1993), 1389-1411.

[2] H.M. Farkas and I. Kra, Riemann surfaces, Grad. Texts in Math., 72, Springer Verlag, Berlin, 1980.

[3] H. Fujimoto, On the number of exceptional values of the Gauss map of minimal surfaces, J. Math. Soc. Japan, 40 (1988), 235-247.

[4] _ Modified defect relations for the Gauss map of minimal surfaces, J. Differential Geom., 29 (1989), 245-262.

[5] F.J. Lopez, Hyperbolic complete minimal surfaces with arbitrary topology, Trans. Amer. Math. Soc., 350(5) (1998), 1977-1990.

[6] W.H. Meeks, The classification of complete minimal surfaces in $\mathbb{R}^{3}$ with total curva-

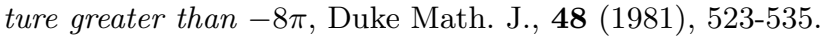


[7] X. Mo and R. Osserman, On the Gauss map and total curvature of complete minimal surfaces and an extension of Fujimoto's theorem, J. Differential Geom., 31 (1990), 343-355.

[8] R. Osserman, A survey of minimal surfaces, Dover Publications, New York, second edition, 1986.

[9] _ Global properties of minimal surfaces in $E^{3}$ and $E^{n}$, Ann. of Math., 80(2) (1964), 340-364.

[10] K. Voss, Über vollständige minimalflächen, L'Enseigment Math., 10 (1964), 316-317.

[11] A. Weitsman and F. Xavier, Some function theoretic properties of the Gauss map for hyperbolic complete minimal surfaces, Michigan Math. J., 34 (1987), 275-283.

[12] F. Xavier, The Gauss map of a complete non flat minimal surface cannot omit 7 points of the sphere, Ann. of Math., 113 (1981), 211-214.

Received September 21, 1998. This research was partially supported by DGICYT grant number PB94-0796.

\author{
UNIVERSIDAD DE GRANADA \\ 18071 GRANADA \\ SPAIN \\ E-mail address: fjlopez@goliat.ugr.es \\ UNIVERSIDAD DE GRANADA \\ 18071 GRANADA \\ SPAIN \\ E-mail address: fmartin@goliat.ugr.es
}

